likely when turning or spinning, and on sloped terrain with passengers. Backward rollovers were most likely with passengers. For those ejected, patients falling/ejected to the rear had the lowest Glasgow Coma Scale (GCS) scores. Patients who jumped off or were ejected off the side had the highest extremity injury scores. The greater the ATV speed, the lower were the patient's GCS scores. 183 ATV crash videos have been collected and characterised to date. Although some ATV crash circumstances were overrepresented in the videos, most demonstrated mechanisms that were seen in actual trauma patients.

Significance/Contribution to the Field Evaluation of crash videos from social media sites may shed light on injury mechanisms. Analysis of these videos yields significant details that are not available through other sources.

\section{A PICTURE IS WORTH A THOUSAND WORDS: UTILISING SOCIAL MEDIA TO BETTER UNDERSTAND ALL-TERRAIN VEHICLE CRASH MECHANISMS OF REAL PATIENTS}

doi:10.1136/injuryprev-2012-040590s.9

${ }^{1} \mathrm{C}$ Jennissen, ${ }^{1} \mathrm{M}$ Price, ${ }^{1,2} \mathrm{~K}$ Harland, ${ }^{1} \mathrm{G}$ Denning. ${ }^{1}$ Department of Emergency Medicine, University of lowa Carver College of Medicine, lowa City, lowa, USA, ${ }^{2}$ Injury Prevention Research Center, College of Public Health, University of lowa, lowa City, lowa, USA

Background Most surveillance sources provide limited data on the sequence of events during ATV crashes. This limitation provides the rationale to investigate less traditional sources such as social media sites where video sharing has become very popular.

Aims/Objectives/Purpose To better understand the mechanisms and contributing factors of ATV-related injuries.

Methods A review of ATV-related injuries at the University of Iowa from 2002 to 2009 was performed and crash mechanisms were characterised. A retrospective search of ATV crash videos posted on YouTube was performed. Videos were compiled and analysed.

Results/0utcome 345 ATV trauma patients were identified. Rollovers (42\%), striking an object (20\%), and ejection/fall (13\%) were the most common mechanisms of injury. The victim was struck by their ATV in 21\% and pinned in $9 \%$. Rollovers were more 